\title{
Matrix Computations Using Quasirandom Sequences
}

\author{
Michael Mascagni ${ }^{1}$ and Aneta Karaivanova ${ }^{1,2}$ \\ 1 Department of Computer Science, Florida State University \\ 203 Love Building, Tallahassee, FL 32306-4530, USA \\ mascagni@cs.fsu.edu \\ http://www.cs.fsu.edu/ mascagni \\ 2 Central Laboratory for Parallel Processing, Bulgarian Academy of Sciences \\ Acad. G. Bonchev St.,bl. 25 A, 1113, Sofia, Bulgaria \\ aneta@scri.fsu.edu
}

\begin{abstract}
The convergence of Monte Carlo method for numerical integration can often be improved by replacing pseudorandom numbers (PRNs) with more uniformly distributed numbers known as quasirandom numbers(QRNs). Standard Monte Carlo methods use pseudorandom sequences and provide a convergence rate of $O\left(N^{-1 / 2}\right)$ using $N$ samples. Quasi-Monte Carlo methods use quasirandom sequences with the resulting convergence rate for numerical integration as good as $\left.O\left((\log N)^{k}\right) N^{-1}\right)$.

In this paper we study the possibility of using QRNs for computing matrix-vector products, solving systems of linear algebraic equations and calculating the extreme eigenvalues of matrices. Several algorithms using the same Markov chains with different random variables are described. We have shown, theoretically and through numerical tests, that the use of quasirandom sequences improves both the magnitude of the error and the convergence rate of the corresponding Monte Carlo methods. Numerical tests are performed on sparse matrices using PRNs and Soboĺ, Halton, and Faure QRNs.
\end{abstract}

\section{Introduction}

Monte Carlo methods (MCMs) are based on the simulation of stochastic processes whose expected values are equal to computationally interesting quantities. Despite the universality of MCMs, a serious drawback is their slow convergence, which is based on the $O\left(N^{-1 / 2}\right)$ behavior of the size of statistical sampling errors. This represents a great opportunity for researchers in computational science. Even modest improvements in the MCM can have substantial impact on the efficiency and range of applicability for MCM. Much of the effort in the development of Monte Carlo methods has been in construction of variance reduction methods which speed up the computation by reducing the constant in front of the $O\left(N^{-1 / 2}\right)$. An alternative approach to acceleration is to change the choice of sequence and hence improve the behavior with $N$. Quasi-Monte Carlo

L. Vulkov, J. Waśniewski, and P. Yalamov (Eds.): NAA 2000, LNCS 1988, pp. 552-559, 2001.

(C) Springer-Verlag Berlin Heidelberg 2001 
methods (QMCMs) use quasirandom (also known as low-discrepancy) sequences instead of pseudorandom sequences.

QRNs are constructed to minimize a measure of their deviation from uniformity called discrepancy. There are many different discrepancies, but let us consider the most common, the star discrepancy. Let us define the star discrepancy of a one-dimensional point set, $\left\{x_{n}\right\}_{n=1}^{N}$, by

$$
D^{\star}=D^{\star}\left(x_{1}, \ldots, x_{N}\right)=\sup _{0 \leq u \leq 1}\left|\frac{1}{N} \sum_{n=1}^{N} \chi_{[0, u)}\left(x_{n}\right)-u\right|
$$

where $\chi_{[0, u)}$ is the characteristic function of the half open interval $[0, u)$. The mathematical motivation for quasirandom numbers can be found in the classic Monte Carlo application of numerical integration. We detail this for the trivial example of one-dimensional integration for illustrative simplicity.

Theorem (Koksma-Hlawka, [6]): if $f(x)$ has bounded variation, $V(f)$, on $[0,1)$, and $x_{1}, \ldots, x_{N} \in[0,1]$ have star discrepancy $D^{\star}$, then:

$$
\left|\frac{1}{N} \sum_{n=1} N f\left(x_{n}\right)-\int_{0}^{1} f(x) d x\right| \leq V(f) D^{\star},
$$

The star discrepancy of a point set of $N$ truly random numbers in one dimension is $O\left(N^{-1 / 2}(\log \log N)^{1 / 2}\right)$, while the discrepancy of $N$ quasirandom numbers can be as low as $N^{-1}$. ${ }^{1}$ In $s>3$ dimensions it is rigorously known that the discrepancy of a point set with $N$ elements can be no smaller than a constant depending only on $s$ times $N^{-1}(\log N)^{(s-1) / 2}$. This remarkable result of Roth, [10], has motivated mathematicians to seek point sets and sequences with discrepancies as close to this lower bound as possible. Since Roth's remarkable results, there have been many constructions of low discrepancy point sets that have achieved star discrepancies as small as $O\left(N^{-1}(\log N)^{s-1}\right)$. Most notably there are the constructions of Hammersley, Halton, [5], Sobol, [11], Faure, [3], and Niederreiter, [9].

While QRNs do improve the convergence of applications like numerical integration, it is by no means trivial to enhance the convergence of all MCMs. In fact, even with numerical integration, enhanced convergence is by no means assured in all situations with the näive use of quasirandom numbers, $[1,8]$.

In this paper we study the applicability of quasirandom sequences for solving some linear algebra problems. We have already produced encouraging theoretical and empirical results with QMCMs for linear algebra problems and we believe that this initial work can be improved.

\section{Solving Systems of Linear Algebraic Equations via Neumann Series}

Assume that a system of linear algebraic equations (SLAE) can be transformed into the following form: $x=A x+\varphi$, where $A$ is a real square, $n \times n$, matrix,

\footnotetext{
${ }^{1}$ Of course, the $N$ optimal quasirandom points in $[0,1)$ are the obvious: $\frac{1}{(N+1)}, \frac{2}{(N+1)}, \ldots \frac{N}{(N+1)}$.
} 
$x=\left(x_{1}, x_{2}, \ldots, x_{n}\right)^{t}$ is the $1 \times n$ solution vector and $\varphi=\left(\varphi_{1}, \varphi_{2}, \ldots, \varphi_{n}\right)^{t}$ is the given right-hand side vector. ${ }^{2}$ In addition, assume that $A$ satisfies either the condition $\max _{1 \leq i \leq n} \sum_{j=1}^{n}\left|a_{i j}\right|<1$, or, that all the eigenvalues of $A$ lie within the unit circle.

Now consider the sequence $x^{(1)}, x^{(2)}, \ldots$ defined by the following recursion:

$$
x^{(k)}=A x^{(k-1)}+\varphi, \quad k=1,2, \ldots
$$

Given initial vector $x^{(0)}$, the approximate solution to the system $x=A x+\varphi$ can be developed via a truncated Neumann series:

$$
x^{(k)}=\varphi+A \varphi+A^{2} \varphi+\ldots+A^{(k-1)} \varphi+A^{k} x^{(0)}, k>0
$$

with a truncation error of $x^{(k)}-x=A^{k}\left(x^{(0)}-x\right)$.

This iterative process (3) of applying the matrix $A$ repeatedly is the basis for deriving a Monte Carlo approach for this problem.

\section{The Monte Carlo Method}

Consider the problem of evaluating the inner product of a given vector, $g$, with the vector solution of the considered system

$$
(g, x)=\sum_{\alpha=1}^{n} g_{\alpha} x_{\alpha} .
$$

To solve this problem via a MCM (see, for example, [12]) one has to construct a random process with mean equal to the solution of the desired problem. This requires the construction of a finite-state Markov chain. Consider the following Markov chain:

$$
k_{0} \rightarrow k_{1} \rightarrow \ldots \rightarrow k_{i},
$$

where $k_{j}=1,2, \ldots, n$ for $j=1, \ldots, i$ are natural numbers. The rules for constructing the chain (5) are: $P\left(k_{0}=\alpha\right)=p_{\alpha}, P\left(k_{j}=\beta \mid k_{j-1}=\alpha\right)=p_{\alpha \beta}$ where $p_{\alpha}$ is the probability that the chain starts in state $\alpha$ and $p_{\alpha \beta}$ is the transition probability from state $\alpha$ to state $\beta$. Probabilities $p_{\alpha \beta}$ define a transition matrix $P$. We require that $\quad \sum_{\alpha=1}^{n} p_{\alpha}=1, \quad \sum_{\beta=1}^{n} p_{\alpha \beta}=1$ for any $\alpha=1,2, \ldots, n$, and that the distribution $\left(p_{1}, \ldots, p_{n}\right)^{t}$ is permissible to the vector $g$ and similarly the distribution $p_{\alpha \beta}$ is permissible to $A$ [12]. Common constructions are to choose $p_{\alpha \beta}=\frac{\left|a_{\alpha \beta}\right|}{\sum_{\beta}\left|a_{\alpha \beta}\right|}$ for $\alpha, \beta=1,2, \ldots, n$, which corresponds to an importance sampling MCM (MCM with a reduced variance), or to choose $p_{\alpha \beta}=1 / n$ for $\alpha, \beta=1,2, \ldots, n$ which corresponds to standard MCM.

Now define the random variables $\theta[g]$ :

$$
\begin{gathered}
\theta[g] \quad=\frac{g_{k_{0}}}{p_{k_{0}}} \sum_{j=0}^{\infty} W_{j} \varphi_{k_{j}} \\
\text { where } W_{0}=1, \quad W_{j}=W_{j-1} \frac{a_{k_{j-1} k_{j}}}{p_{k_{j-1} k_{j}}},
\end{gathered}
$$

\footnotetext{
${ }^{2}$ If we consider a given system $L x=b$, then it is possible to find a non-singular matrix, $M$, such that $M L=I-A$ and $M b=\varphi$. Thus without loss of generality the system $L x=b$ can always be recast as $x=A x+\varphi$.
} 
It is known [12] that the mathematical expectation $E[\theta[g]]$ of the random variable $\theta[g]$ is:

$$
E[\theta[g]]=(g, x)
$$

The partial sum corresponding to (6) is defined as $\theta_{i}[g]=\frac{g_{k_{0}}}{p_{k_{0}}} \sum_{j=0}^{i} W_{j} \varphi_{k_{j}}$. Thus the Monte Carlo estimate for $(g, x)$ is $(g, x) \approx \frac{1}{N} \sum_{s=1}^{N} \theta_{i}[g]_{s}$, where $N$ is the number of chains and $\theta_{i}[g]_{s}$ is the value of $\theta_{i}[g]$ taken over the $s$-th chain, and a statistical error of size $O\left(\operatorname{Var}\left(\theta_{i}\right)^{1 / 2} N^{-1 / 2}\right)$.

\section{Computing the Extremal Eigenvalues}

Let $A$ be an $n \times n$ large, sparse, matrix. Consider the problem of computing one or more eigenvalues of $A$, i.e., the values of $\lambda$ for which $A u=\lambda u$ holds. Suppose the eigenvalues are ordered $\left|\lambda_{1}\right|>\left|\lambda_{2}\right| \geq \ldots \geq\left|\lambda_{n-1}\right|>\left|\lambda_{n}\right|$. There are two deterministic numerical methods that can efficiently compute only the extremal eigenvalues - the power method and Lanczos-type methods. (Note that, the Lanczos method is applicable to only symmetric eigenproblems, [4]. )

Computational Complexity: If $k$ iterations are required for convergence, the number of arithmethic operations is $O\left(k n^{2}\right)$ for the power method and $O\left(n^{3}+k n^{2}\right)$ for both the inverse and inverse shifted power method.

\section{The Monte Carlo Method}

Consider MCMs based on the power method. When computing eigenvalues, we work with the matrix $A$ and its resolvent matrix $R_{q}=[I-q A]^{-1} \in \mathbb{R}^{n \times n}$. If $|q \lambda|<1, R_{q}$ may be expanded as a series via the binomial theorem:

$$
[I-q A]^{-m}=\sum_{i=1}^{\infty} q^{i} C_{m+i-1}^{i}, \quad|q \lambda|<1 .
$$

The eigenvalues of the matrices $R_{q}$ and $A$ are connected by the equality $\mu=$ $\frac{1}{1-q \lambda}$, and the eigenvectors of the two matrices coincide ${ }^{3}$. Let $f \in \mathbb{R}^{n}, h \in \mathbb{R}^{n}$. Applying the power method, ([2]), leads to the following iterative processes:

$$
\begin{gathered}
\lambda^{(m)}=\frac{\left(h, A^{i} f\right)}{\left(h, A^{i-1} f\right)} \underset{m \rightarrow \infty}{\longrightarrow} \lambda_{\max } \\
\mu^{(m)}=\frac{\left([I-q A]^{-m} f, h\right)}{\left([I-q A]^{-(m-1)} f, h\right)} \underset{m \rightarrow \infty}{\longrightarrow} \mu_{\max }=\frac{1}{1-q \lambda} .
\end{gathered}
$$

Construct the same Markov chain as before with the initial density vector, $p=$ $\left\{p_{\alpha}\right\}_{\alpha=1}^{n}$, and the transition density matrix, $P=\left\{p_{\alpha \beta}\right\}_{\alpha \beta=1}^{n}$. Define the following

${ }^{3}$ If $q>0$, the largest eigenvalue $\mu_{\max }$ of the resolvent matrix corresponds to the largest eigenvalue, $\lambda_{\max }$, of the matrix $A$, but if $q<0$, then $\mu_{\max }$, corresponds to the smallest eigenvalue, $\lambda_{\min }$, of the matrix $A$. 
random variable: $W_{0}=\frac{h_{k_{0}}}{p_{k_{0}}}, W_{j}=W_{j-1} \frac{a_{k_{j-1} k_{j}}}{p_{k_{j-1} k_{j}}}, j=1, \ldots, i$. This has the desired expected values ([2]):

$$
\begin{gathered}
E\left[W_{i} f_{k_{i}}\right]=\left(h, A^{i} f\right), i=1,2, \ldots, \\
E\left[\sum_{i=0}^{\infty} q^{i} C_{i+m-1}^{i} W_{i} f\left(x_{i}\right)\right]=\left(h,[I-q A]^{-m} f\right), m=1,2, \ldots,
\end{gathered}
$$

and allows us to estimate the desired eigenvalues as:

$$
\lambda_{\max } \approx \frac{E\left[W_{i} f_{k_{i}}\right]}{E\left[W_{i-1} f_{k_{i-1}}\right]} .
$$

and

$$
\lambda \approx \frac{1}{q}\left(1-\frac{1}{\mu^{(m)}}\right)=\frac{E\left[\sum_{i=1}^{\infty} q^{i-1} C_{i+m-2}^{i-1} W_{i} f\left(x_{i}\right)\right]}{E\left[\sum_{i=0}^{\infty} q^{i} C_{i+m-1}^{i} W_{i} f\left(x_{i}\right)\right]} .
$$

We remark that in (10) the length of the Markov chain, $l$, is equal to the number of iterations, $i$, in the power method. However in (11) the length of the Markov chain is equal to the number of terms in truncated series for the resolvent matrix. In this second case the parameter $m$ corresponds to the number of iterations.

Table 1. Monte Carlo estimations using PRNs and QRN sequences for the dominant eigenvalue of matrices of size 128 and 2000

\begin{tabular}{||l||r|r|r|r||}
\hline \hline & $P R N$ & $Q R N($ Faur $)$ & $Q R N($ Sobol $)$ & $Q R N($ Halton $)$ \\
\hline $\begin{array}{l}\text { Estimated } \\
\lambda 128_{\max }\end{array}$ & 61.2851 & 63.0789 & 63.5916 & 65.1777 \\
\hline $\begin{array}{l}\text { Relative } \\
\text { Error }\end{array}$ & 0.0424 & 0.0143 & 0.0063 & 0.0184 \\
\hline \hline $\begin{array}{l}\text { Estimated } \\
\lambda 2000_{\max }\end{array}$ & 58.8838 & 62.7721 & 65.2831 & 65.377 \\
\hline $\begin{array}{l}\text { Relative } \\
\text { Error }\end{array}$ & 0.0799 & 0.01918 & 0.0200 & 0.0215 \\
\hline \hline
\end{tabular}

\section{Quasi-Monte Carlo Methods for Matrix Computations}

Recall that power method iterations are based on computing $h^{T} A^{i} f$ (see (8) and (9)). Even if we are interested in evaluating the inner product (4), substituting $x$ with $x^{(k)}$ from (3) will give $(g, x) \approx g^{T} \varphi+g^{T} A \varphi+g^{T} A^{2} \varphi+\ldots+g^{T} A^{(k-1)} \varphi+$ $g^{T} A^{k} x^{(0)}, k>0$. Define the sets $G=[0, n)$ and $G_{i}=[i-1, i), i=1, \ldots, n$, and likewize define the piecewise continous functions $f(x)=f_{i}, x \in G_{i}, i=1, \ldots, n$, $a(x, y)=a_{i j}, x \in G_{i}, y \in G_{j}, i, j=1, \ldots, n$ and $h(x)=h_{i}, x \in G_{i}, i=$ $1, \ldots, n$. 
Computing $h^{T} A^{i} f$ is equivalent to computing an $(i+1)$-dimensional integral. Thus we may analyze using QRNs in this case with bounds from numerical integration. We do not know $A^{i}$ explicitly, but we do know $A$ and can use a random walk on the elements of the matrix to compute approximately $h^{T} A^{i} f$.

\section{Relative Errors in Computing $h^{\top} A^{k^{k}}$}

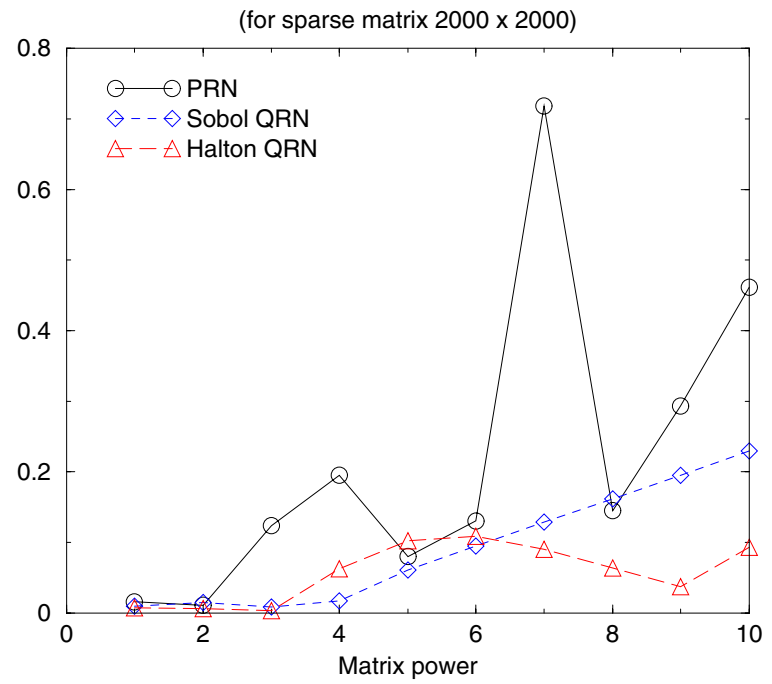

Fig. 1. Relative errors in computing $h^{T} A^{k} f$ for $k=1,2, \ldots, 10$ for a sparse matrix $2000 \times 2000$. The corresponding Markov chains are realized using PRN, Soboĺ and Halton sequences

Consider $h^{T} A^{i} f$ and an $(i+1)$-dimensional QRN sequence. Normalizing $A$ with $\frac{1}{n}$, and $h$ and $f$ with $\frac{1}{\sqrt{n}}$, we have the following error bound (for proof see [7]):

$$
\left|h_{N}^{T} A_{N}^{l} f_{N}-\frac{1}{N} \sum_{s=1}^{N} h\left(x_{s}\right) a\left(x_{s}, y_{s}\right) \ldots a\left(z_{s}, w_{s}\right) f\left(w_{s}\right)\right| \leq|h|^{T}|A|^{l}|f| D_{N}^{*} .
$$

If $\mathrm{A}$ is a general sparse matrix with $d$ nonzero elements per row, and $d \ll n$, then importance sampling method can be used; the normalizing factors in the error bound (3) are then $1 / d$ for the matrix and $\frac{1}{\sqrt{(} n)}$ for the vectors.

\section{Numerical Results}

Why are we interested in quasi-MCMs for the eigenvalue problem? Because the computational complexity of QMCMs is bounded by $O(l N)$ where $N$ is the number of chains, and $l$ is the mathematical expectation of the length of the Markov 
chains, both of which are independent of matrix size $n$. This makes QMCMs very efficient for large, sparse, problems, for which deterministic methods are not computationally efficient.

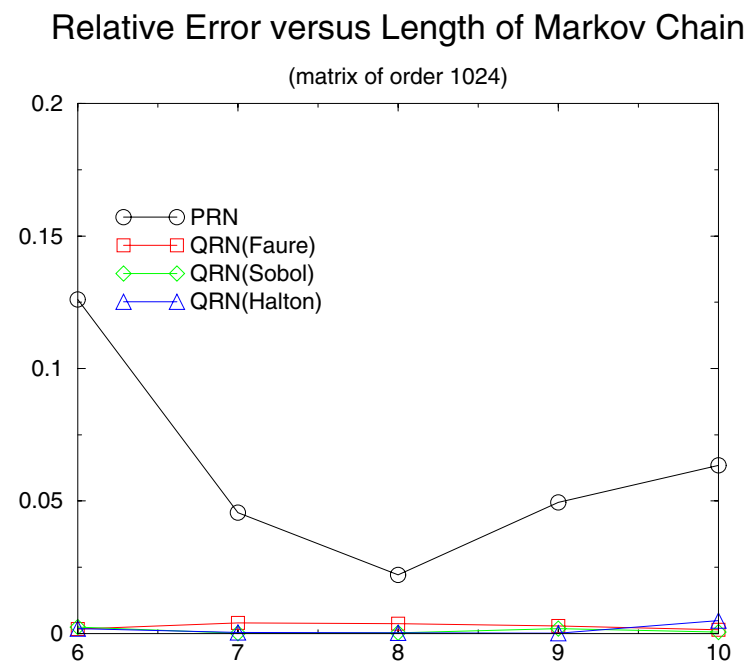

Fig. 2. Relative errors in computing $\lambda_{\max }$ using different length of Markov chains for a sparse matrix $1024 \times 1024$. The random walks are realized using PRN, Faure, Sobol and Halton sequences

Numerical tests were performed on general sparse matrices using PRNs and Sobol, Halton and Faure QRNs. The relative errors in computing $h^{T} A^{k} f$ with $A$ a sparse matrix of order 2000 and $h=f=(1,1, \ldots, 1)$, are presented in Figure 1. The results confirm that the QRNs produce higher precision results than PRNs. The more important fact is the smoothness of the quasirandom "iterations" with $k$. This is important because these eigenvalue algorithms compute a Raleigh quotient which requires the division of values from consecutive iterations.

The estimated $\lambda_{\max }$ and the corresponding relative errors using MCM and QMCM are presented in Table 1. The exact value of $\lambda_{\max }$ for all test matrices is 64.0000153. The results show improvement of the accuracy. Numerical experiments using resolvent MCM and resolvent QMCM have been also performed the relative errors in computing $\lambda_{\max }$ using Markov chains with different lengths are presented in Figures 2 and 3.

\section{Acknowledgements}

This paper is based upon work supported by the North Atlantic Treaty Organization under a Grant awarded in 1999. 


\section{Relative Error versus Length of Markov Chain}

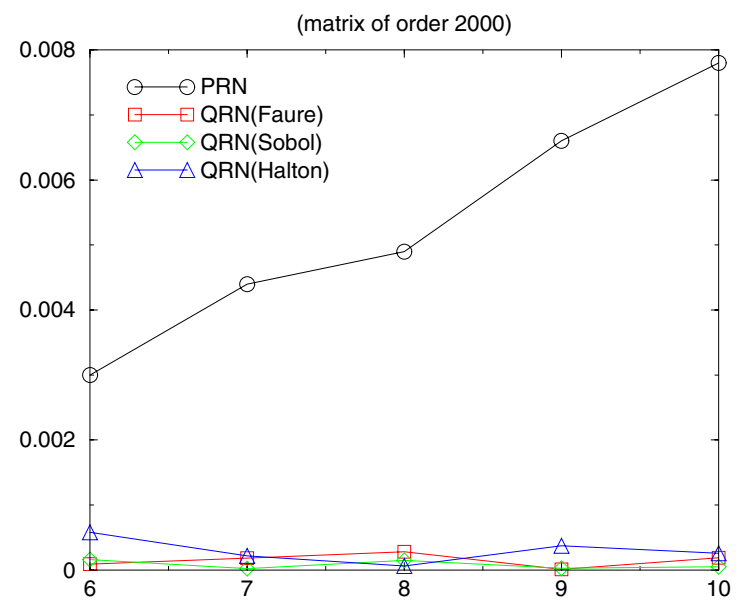

Fig. 3. Relative errors in computing $\lambda_{\max }$ using different length of Markov chains for a sparse matrix $2000 \times 2000$. The random walks are realized using PRN, Faure, Sobol and Halton sequences

\section{References}

1. R. E. Caflisch, "Monte Carlo and quasi-Monte Carlo methods," Acta Numerica, 7: $1-49,1998.553$

2. I. Dimov, A. Karaivanova, "Parallel computations of eigenvalues based on a Monte Carlo approach," J. of MC Methods and Appl., 4, Num.1: 33-52, 1998. 555,556

3. H. FAure, "Discrépance de suites associées à un système de numération (en dimension s)," Acta Arithmetica, XLI: 337-351, 1992. 553

4. G. H. Golub, C. F. VAn LoAn, "Matrix computations", The Johns Hopkins Univ. Press, Baltimore, 1996. 555

5. J. H. Halton, "On the efficiency of certain quasi-random sequences of points in evaluating multi-dimensional integrals," Numer. Math., 2: 84-90, 1960. 553

6. J. F. KoKsma, "Een algemeene stelling uit de theorie der gelijkmatige verdeeling modulo 1," Mathematica B (Zutphen), 11: 7-11, 1942/43. 553

7. M. Mascagni, A. Karaivanova, "Are Quasirandom Numbers Good for Anything Besides Integration?" to appear in Proc. of Advances in Reactor Physics and Mathematics and Computation into the Next Millennium (PHYSOR2000), 2000. 557

8. B. Moskowitz, R. E. Caflisch, "Smoothness and dimension reduction in quasiMonte Carlo methods", J. Math. Comput. Modeling, 23: 37-54, 1996. 553

9. H. NiederReiter, Random number generation and quasi-Monte Carlo methods, SIAM: Philadelphia, 1992. 553

10. K. F. Rотн, "On irregularities of distribution," Mathematika, 1: 73-79, 1954. 553

11. I. M. SoвoĹ, "The distribution of points in a cube and approximate evaluation of integrals," Zh. Vychisl. Mat. Mat. Fiz., 7: 784-802, 1967. 553

12. I. M. Sobó́ Monte Carlo numerical methods, Nauka, Moscow, 1973. 554, 555 